\title{
Folding model analysis of proton scattering from mirror nuclei ${ }^{18} \mathrm{Ne}$ and ${ }^{18} \mathrm{O}$
}

\author{
D. Gupta*†, D. N. Basu \\ Variable Energy Cyclotron Centre, 1/AF Bidhan Nagar, Kolkata 700 064, India
}

(November 5, 2018)

\begin{abstract}
The elastic and inelastic scattering of protons from mirror nuclei ${ }^{18} \mathrm{Ne}$ and ${ }^{18} \mathrm{O}$ are studied in a folding model approach. For comparison, two different effective interactions are folded with Hartree-Fock densities to obtain the nuclear interaction potentials. Both of them provide equivalent descriptions to the data and the deformation parameters extracted from inelastic scattering are reasonable. The density dependence parameters obtained from nuclear matter calculations and used for present analysis also provide a good estimate for the nuclear mean free path. The present formalism unifies radioactivity, nuclear matter and nuclear scattering.
\end{abstract}

Keywords: Elastic and Inelastic Proton Scattering; Effective Interaction; Folding Model; SBM; DDM3Y; Mirror Nuclei

PACS numbers: 25.40.Cm, 25.40.Ep, 21.30.Fe, 25.60.-t

\section{INTRODUCTION}

Proton scattering has been widely used as a means to study both collective and microscopic aspects of nuclear structure $[1,2]$. The study is consistent only if a well-defined effective nucleon-nucleon (NN) interaction is applied in the analysis. Also, with the advent of radioactive nuclear beams there is constant enhancement of our knowledge frontiers on the structure and reaction dynamics of the known stable nuclei as well as their less known unstable counterparts [3,4]. This rapidly developing field provides a testing ground for different nuclear reaction theories and effective interactions. Scattering involving ${ }^{18} \mathrm{Ne}$ and ${ }^{18} \mathrm{O}$ are interesting because not only are they mirror nuclei, but also ${ }^{18} \mathrm{O}$ is a stable nucleus while ${ }^{18} \mathrm{Ne}$ is its radioactive counterpart.

In this work, proton scattering on ${ }^{18} \mathrm{Ne}$ and ${ }^{18} \mathrm{O}$ has been studied at low energies $(<100 \mathrm{MeV} / \mathrm{A})[5,6]$ in a folding model approach. The folding model is well known as a powerful tool for analyzing nucleus-nucleus scattering data at relatively low incident energies [4,7-9]. It directly links the density profile of the nucleus with the scattering cross sections and is thus very appropriate for studying nuclei, especially those with exotic matter distributions. A semi-microscopic analysis in the optical model $(\mathrm{OM})$ framework is carried out. In the DWBA calculations of nuclear excitation, with transferred angular momentum $l$, the form factors are obtained by taking the derivatives of the potentials used.

\section{THEORETICAL FORMULATION}

The nucleon-nucleus potential can be obtained by single folding the density distribution of the nucleus with the nucleon-nucleon effective interaction [10] as,

$$
U\left(\overrightarrow{r_{1}}\right)=\int \rho_{2}\left(\overrightarrow{r_{2}}\right) v_{\mathrm{NN}}\left(\left|\overrightarrow{r_{1}}-\overrightarrow{r_{2}}\right|\right) d^{3} \overrightarrow{r_{2}}
$$

where $\rho_{2}\left(\overrightarrow{r_{2}}\right)$ is density of the nucleus at $\overrightarrow{r_{2}}$ and $v_{\mathrm{NN}}$ is the effective interaction between two nucleons at the sites $\overrightarrow{r_{1}}$ and $\overrightarrow{r_{2}}$. Two different forms of effective interactions have been employed in this work. We perform a comparative study between the Modified Seyler-Blanchard (SBM) and density dependent M3Y (DDM3Y) effective NN interactions.

The finite range, density, momentum and isospin dependent effective interaction SBM has different strengths for pp (or nn) and pn interactions and its form is [11],

$$
v\left(r=\left|\overrightarrow{r_{1}}-\overrightarrow{r_{2}}\right|, p, \rho\right)=-C_{l, u} \frac{e^{-r / a}}{r / a}\left[1-\frac{p^{2}}{b^{2}}-d^{2}\left(\rho_{1}+\rho_{2}\right)^{n}\right]
$$

*E-mail:dgupta@veccal.ernet.in

${ }^{\dagger}$ Present Address: Institut de Physique Nucleaire, 91406 Orsay Cedex, France 
where, the subscripts ' $l$ ' and ' $u$ ' refer to like-pair (nn or pp) and unlike-pair (np) interactions, respectively. Here ' $a$ ' is the range of the two-body interaction, ' $b$ ' is a measure of the strength of repulsion with relative momentum ' $p$ ', while ' $d$ ' and ' $n$ ' are two parameters determining the strength of density dependence. $\rho_{1}\left(\overrightarrow{r_{1}}\right)$ and $\rho_{2}\left(\overrightarrow{r_{2}}\right)$ are densities at the sites of the two interacting nucleons. The values of parameters $n, C_{\mathrm{l}}, C_{\mathrm{u}}, a, b, d$ are given in Table 1 . These constants are found to reproduce the bulk properties of nuclear matter and of finite nuclei $[11,12]$ and are known also to explain the $\mathrm{p}+{ }^{4,6,8} \mathrm{He},{ }^{6,7,9,11} \mathrm{Li}$ scattering data successfully $[2,4,13-16]$. The parameters are determined without exchange effects and thus they contain the effect indirectly though in a very approximate way.

The finite range M3Y effective interaction $v(r)$ appearing in Eqn. 1 is given by [17]

$$
v(r)=7999 \frac{e^{-4 r}}{4 r}-2134 \frac{e^{-2.5 r}}{2.5 r}
$$

This interaction is based upon a realistic G-matrix, which was constructed in an oscillator representation. Effectively it is an average over a range of nuclear densities as well as energies and therefore the M3Y has no explicit density or energy dependence. The only energy dependent effect that arises from its use is a rather weak one contained in an approximate treatment of single-nucleon knock-on exchange. The density and energy averages are adequate for the real part of the optical potential for heavy ions at lower energies. Although, it is important to consider the density and energy dependence explicitly for scattering at higher energies, where the effects of a nuclear rainbow are seen and hence the scattering becomes sensitive to the potential at small radii. Such cases were studied introducing suitable and semirealistic explicit density dependence $[18,19]$ into the M3Y interaction which was then called the DDM3Y and was very successful for interpreting consistently the high energy elastic $\alpha$ and heavy ion scattering data. The present calculations use the density dependent M3Y effective interaction (DDM3Y) supplemented by a zero-range pseudo potential. In DDM3Y, the effective nucleon-nucleon interaction $v(r)$ is assumed to be density and energy dependent and therefore becomes functions of density and energy and is given by

$$
v(r, \rho, E)=t^{\mathrm{M} 3 \mathrm{Y}}(r, E) g(\rho, E)
$$

where $t^{\mathrm{M} 3 \mathrm{Y}}$ is the same M3Y interaction given by Eqn. 3 but supplemented by a zero range pseudo-potential [18]

$$
t^{\mathrm{M} 3 \mathrm{Y}}=7999 \frac{e^{-4 r}}{4 r}-2134 \frac{e^{-2.5 r}}{2.5 r}+J_{00}(E) \delta(r)
$$

where the zero-range pseudo-potential representing the single-nucleon exchange term is given by

$$
J_{00}(E)=-276(1-0.005 E / A) \mathrm{MeV} \cdot \mathrm{fm}^{3}
$$

and the density dependent part has been taken to be [19]

$$
g(\rho, E)=C\left(1-\beta(E) \rho^{2 / 3}\right)
$$

which takes care of the higher order exchange effects and the Pauli blocking effects. $E / A$ is energy per nucleon. The constants of this interaction $C$ and $\beta$ when used in single folding model description, can be determined by nuclear matter calculations [20] as 2.07 and $1.624 \mathrm{fm}^{2}$ respectively. The density dependence parameter $\beta$ has the dimension of cross-section. The term $\left(1-\beta \rho^{2 / 3}\right)$ reduces the strength of the interaction and changes sign at high densities making it repulsive. This is a direct consequence of the Pauli blocking effect. Thus $\left(1-\beta \rho^{2 / 3}\right)$ represents the probability of non-interaction arising due to collision probability $\beta \rho^{2 / 3}$ of a nucleon in nuclear medium of density $\rho$. The parameter $\beta$ can be identified as the 'in medium' effective nucleon-nucleon interaction cross section $\sigma_{0}$. This value of $\beta$ along with nucleonic density of infinite nuclear matter $\rho_{0}$ can also provide the nuclear mean free path $\lambda=\left(\rho_{0} \sigma_{0}\right)^{-1}$.

\section{CALCULATION AND ANALYSIS}

The nuclear ground state densities have been calculated in the framework of spherical Hartree Fock plus BCS calculations in co-ordinate space using two different parameter sets of the Skyrme interactions given by [21]

$$
v_{\text {central }}\left(\mathbf{r}_{\mathbf{1}}, \mathbf{r}_{\mathbf{2}}\right)=t_{0}\left(1+x_{0} \mathbf{P}_{\sigma}\right) \delta(\mathbf{r})+t_{1}\left(1+x_{1} \mathbf{P}_{\sigma}\right)\left[\delta(\mathbf{r}) \mathbf{k}^{\mathbf{2}}+\mathbf{k}^{\mathbf{2}^{2}} \delta(\mathbf{r})\right]+t_{2}\left(1+x_{2} \mathbf{P}_{\sigma}\right) \mathbf{k}^{\prime} \delta(\mathbf{r}) \mathbf{k}+t_{3}\left(1+x_{3} \mathbf{P}_{\sigma}\right) \rho^{\alpha}(\mathbf{R}) \delta(\mathbf{r})
$$

There are negligible differences in the ground state densities of the nuclei with the two different parameter sets (for SIII [22] and SkM* [21]) provided in Table 2. We show $\mathrm{SkM}^{*}$ parameterization for the ${ }^{18} \mathrm{O}$ ground state density (Fig. 
1) and used it for calculations of the single folding potential and form factor. We carried out the same procedure for ${ }^{18} \mathrm{Ne}$ and for $\mathrm{SkM}^{*}$ the binding energies per nucleon are in good agreement (within $0.1 \mathrm{MeV}$ ) with the experimental values for both the nuclei.

The potentials used while calculating phenomenological best fits [6,23] have the following form,

$$
V_{\text {pheno }}(r)=-V_{o} f_{o}(r)-i W_{v} f_{v}(r)+4 i a_{s} W_{s}(d / d r) f_{s}(r)+2\left(\hbar / m_{\pi} c\right)^{2} V_{s . o} 1 / r(d / d r) f_{s . o}(r)(\mathbf{L} . \mathbf{S})+V_{\text {coul }}
$$

where, $f_{x}(r)=\left[1+\exp \left(\frac{r-R_{x}}{a_{x}}\right)\right]^{-1}, R_{x}=r_{x} A^{1 / 3}$ and $x=o, v$, s, s.o. The subscripts $o, v$, s, s.o denote real, volume imaginary, surface imaginary and spin-orbit respectively and $V_{o}, W_{v}\left(W_{s}\right)$ and $V_{s . o}$ are the strengths of the real, volume (surface) imaginary and spin-orbit potentials respectively. $V_{\text {coul }}$ is the Coulomb potential of a uniformly charged sphere of radius $1.20 \mathrm{~A}^{1 / 3}$.

In semi-microscopic analysis both the volume real $(V)$ and volume imaginary $(W)$ parts of the potentials (generated microscopically by folding model) are assumed to have the same shape, as in Ref. [4], i.e. $V_{\text {micro }}(r)=V+i W=\left(N_{\mathrm{R}}\right.$ $\left.+i N_{\mathrm{I}}\right) U\left(r_{1}\right)$ where, $N_{\mathrm{R}}$ and $N_{\mathrm{I}}$ are the renormalization factors for real and imaginary parts respectively [9]. In Fig. 2 the p $+{ }^{18} \mathrm{O}$ renormalized real folded potentials (employing the SBM and DDM3Y interactions) are shown at $E$ $=24.5 \mathrm{~A} \mathrm{MeV}$, along with the best fit phenomenological real part. Thus the potentials for elastic scattering analysis include real and volume imaginary terms (folded potentials) and also surface imaginary and spin-orbit terms (best fit phenomenological potentials).

For each angular distribution, best fits are obtained by minimizing $\chi^{2} / \mathrm{N}$, where $\chi^{2}=\sum_{k=1}^{\mathrm{N}}\left[\frac{\sigma_{t h}\left(\theta_{k}\right)-\sigma_{e x}\left(\theta_{k}\right)}{\Delta \sigma_{e x}\left(\theta_{k}\right)}\right]^{2}$, where $\sigma_{t h}$ and $\sigma_{e x}$ are the theoretical and experimental cross sections respectively, at angle $\theta_{k}, \Delta \sigma_{e x}$ is the experimental error and $\mathrm{N}$ is the number of data points. The potentials for elastic scattering analysis are subsequently used in the DWBA calculations of inelastic scattering with transferred angular momentum $l$. The calculations are performed using the code DWUCK4 [24]. The derivative of the potentials $\left(\delta \frac{d V}{d r}\right)$ are used as the form factors. The microscopic real and imaginary form factors have the same shape with strengths $N_{\mathrm{R}}^{\mathrm{FF}}$ and $N_{\mathrm{I}}^{\mathrm{FF}}$ respectively, where $N_{\mathrm{R}, \mathrm{I}}^{\mathrm{FF}}=$ $N_{\mathrm{R}, \mathrm{I}} r_{\mathrm{rms}}^{V}$, where the radius parameter $r_{\mathrm{rms}}^{V}$ is the rms radius of the folded potential. In addition, form factors derived from phenomenological surface imaginary and spin-orbit potentials are included. The deformation parameters $\delta$ are determined by fitting the inelastic scattering angular distribution. The renormalizations required for the potentials are reminiscent of those for deuteron and ${ }^{6} \mathrm{Li}$ scattering and it may be surmised that it is for the same reasons; weak binding and ease of breakup and other reaction channels. Table 1 gives the parameters of the interactions used here. Both interactions provide incompressibility of $\sim 300 \mathrm{MeV}$ for spin and isospin symmetric cold infinite nuclear matter. Moreover, in case of DDM3Y, $\beta$ value of $1.624 \mathrm{fm}^{2}$ obtained from nuclear matter calculations, along with nucleonic density of $0.16 \mathrm{fm}^{-3}$ provides a mean free path of about $4 \mathrm{fm}$ which is in excellent agreement with other theoretical estimates [25]. Table 3 gives the phenomenological best fit optical model parameters while Table 4 gives the renormalization factors, $\delta$ values, $\chi^{2} / \mathrm{N}$ for the folding model analysis.

The relationship between the reduced electric quadrupole transition rate $B(E 2)$ for the ground state to the $2^{+}$state in units of $e^{2} \mathrm{fm}^{4}$ and the quadrupole deformation parameter $\delta$ is given by [26]

$$
\delta\left(1+0.16 \delta+0.20 \delta^{2}+\ldots\right)=4 \pi B(E 2)^{1 / 2} /\left(3 Z R^{2}\right)
$$

where $R=1.2 A^{1 / 3} \mathrm{fm}$ and $Z$ is the atomic number. The quadrupole deformations listed in reference [26] were obtained by using Eqn. 10 but keeping only the terms up to first order in $\delta$. We have recalculated these values by keeping terms up to third order in $\delta$. The recalculated quadrupole deformations thus obtained from the experimental $B(E 2)$ values listed in reference [26] are 0.33078 and 0.59284 for ${ }^{18} \mathrm{O}$ and ${ }^{18} \mathrm{Ne}$ respectively. As can be seen from Table 4, the quadrupole deformation obtained from the present analysis for ${ }^{18} \mathrm{O}$ is in excellent agreement while that for ${ }^{18} \mathrm{Ne}$ is significantly underestimated due to lack of experimental data at forward angles. The inelastic scattering is more sensitive at forward angles due to its relative purity compared to data corresponding to backward angular range where other non-elastic processes also contribute. The quality of elastic as well as the inelastic fits deteriorate at backward angles and similar deterioration of fits are also seen for proton scattering from other nuclei [10,27]. The reason for this is probably that the full cross section is ascribed to potential scattering while quasi-compound-nucleus formation feeds back into the elastic channel and whose energy-dependence is controlled by barrier-top resonances. The backward angular range classically corresponds to smaller impact parameters. This fact suggests higher compound nuclear formation probabilities at backward angles while those at forward angles are expected to be negligibly small. Since relative contributions of compound elastic and direct elastic are not disentangled, $\chi^{2}$ was calculated only upto about $90^{\circ}$ in center of mass during fitting the ${ }^{18} \mathrm{O}$ data.

It may be noted that since we could not acquire the experimental data for ${ }^{18} \mathrm{O}$, they have been read quite accurately from the plots in the original paper [6] and a 5\% uniform error has been assumed. The technique used for extracting the data from plots along with relevant co-ordinate transformations are described in the appendix. 


\section{CONCLUSION}

In the present study we find that the parametrized SBM effective interaction, and the realistic DDM3Y effective interaction obtained from sophisticated G-matrix calculations provide equivalent descriptions for the elastic and inelastic scattering of protons from the mirror nuclei ${ }^{18} \mathrm{Ne}$ and ${ }^{18} \mathrm{O}$ (Fig. 3, 4). The values of the deformation parameters have been extracted from the calculations for these nuclei. Even though the analysis reported here is quite detailed, measurements at higher energies may still be useful in distinguishing various effective interactions. The lack of sensitivity is due to the fact that at such energies, nuclear densities primarily probed are near the surface, which makes the density dependent effects less realizable. The form of density dependence used here is more physical as compared to other forms. The parameter $\beta$ can be interpreted as 'in medium' nucleon-nucleon interaction cross section while $\left(1-\beta \rho^{2 / 3}\right)$ as the non-interaction probability arising due to higher order exchange and Pauli blocking effects.

In summary, a consistent folding model analysis of proton scattering on $\mathrm{A}=18$ nuclei is carried out using two different effective NN interactions. The conventional way of generating the form factors is followed, that is, by taking the derivatives of the potentials (microscopic real and imaginary as well as phenomenological surface imaginary and spin-orbit potentials). Deformation parameters $(\delta)$ are extracted from the analyses. The results obtained for the deformation parameter are in good agreement with the available results. The density dependence parameter obtained from nuclear matter calculations, which has been used in the single folding model description for the analysis of elastic and inelastic scattering of protons, also provides excellent estimate for nuclear mean free path. We know that the DDM3Y effective interaction, has profound theoretical basis. It provides unified description of cluster radioactivity, scatterings of $\alpha$ and heavy ion when used in a double folding model, and nuclear matter when used in a single folding model. We find that it also provides reasonable description for elastic and inelastic scattering of protons.

The authors gratefully acknowledge L. A. Riley for sending the experimental data in a tabular form.

Table 1:

Parameters of the SBM and DDM3Y interactions

\begin{tabular}{|c|c|c|c|c|c|c|}
\hline$\overline{\mathrm{SBM}}$ & $n$ & $C_{l}(\mathrm{MeV})$ & $\overline{C_{u}(\mathrm{MeV})}$ & $\overline{a(\mathrm{fm})}$ & $\overline{b(\mathrm{MeV} / \mathrm{c})}$ & $\overline{d e(\mathrm{fm})}$ \\
\hline & $2 / 3$ & 215.7 & 669.3 & 0.554 & 668.7 & 0.813 \\
\hline \multirow[t]{2}{*}{ DDM3Y } & $n$ & $C$ & $\alpha\left(\mathrm{MeV}^{-1}\right)$ & $\beta\left(\mathrm{fm}^{2}\right)$ & & \\
\hline & $2 / 3$ & 2.07 & 0.005 & 1.624 & & \\
\hline
\end{tabular}

Table 2:

Parameter sets of the Skyrme interactions

\begin{tabular}{|c|c|c|c|c|c|c|c|c|c|c|}
\hline Interaction & $t_{0}$ & $t_{1}$ & $t_{2}$ & $t_{3}$ & $t_{4}$ & $x_{0}$ & $x_{1}$ & $x_{2}$ & $x_{3}$ & $\alpha$ \\
\hline SIII & -1128.75 & 395.0 & -95.0 & 14000.0 & 120.0 & 0.45 & 0.0 & 0.0 & 1.0 & 1 \\
\hline $\mathrm{SkM}^{*}$ & -2645.0 & 410.0 & -135.0 & 15595.0 & 130.0 & 0.09 & 0.0 & 0.0 & 0.0 & $1 / 6$ \\
\hline
\end{tabular}

Table 3:

Phenomenological potential parameters used in $\mathrm{p}+{ }^{18} \mathrm{Ne}$ and ${ }^{18} \mathrm{O}$ scattering

\begin{tabular}{|c|c|c|c|c|c|c|c|c|c|c|c|c|c|c|c|c|}
\hline Nucleus & $\begin{array}{c}E / A \\
(\mathrm{MeV})\end{array}$ & $\begin{array}{c}V_{o} \\
(\mathrm{MeV})\end{array}$ & $\begin{array}{l}r_{o} \\
(\mathrm{fm})\end{array}$ & $\begin{array}{c}a_{o} \\
(\mathrm{fm})\end{array}$ & $\begin{array}{c}W_{v} \\
(\mathrm{MeV})\end{array}$ & $\begin{array}{c}r_{v} \\
(\mathrm{fm})\end{array}$ & $\begin{array}{c}a_{v} \\
(\mathrm{fm})\end{array}$ & $\begin{array}{c}W_{s} \\
(\mathrm{MeV})\end{array}$ & $\begin{array}{c}r_{s} \\
(\mathrm{fm})\end{array}$ & $\begin{array}{c}a_{s} \\
(\mathrm{fm})\end{array}$ & $\begin{array}{c}V_{s . o} \\
(\mathrm{MeV})\end{array}$ & $\begin{array}{l}r_{s . o} \\
(\mathrm{fm})\end{array}$ & $\begin{array}{l}a_{s . o} \\
(\mathrm{fm})\end{array}$ & $\chi_{\mathrm{el}}^{2} / \mathrm{N}$ & $\begin{array}{c}J / A \\
\left(\mathrm{MeV} \mathrm{fm}^{3}\right)\end{array}$ & Ref. \\
\hline${ }^{18} \mathrm{Ne}$ & 30.0 & 40.00 & 1.100 & 0.730 & & & & 7.00 & 1.380 & 0.600 & 7.80 & 1.090 & 0.740 & 5.265 & -364.7 & [6] \\
\hline${ }^{18} \mathrm{O}$ & 24.5 & 48.57 & 1.163 & 0.780 & 2.266 & 1.169 & 0.690 & 5.77 & 1.169 & 0.690 & 5.90 & 0.882 & 0.630 & 6.896 & -527.0 & 23 \\
\hline
\end{tabular}


Table 4:

Renormalizations of SBM and DDM3Y folded potentials and form factors for $\mathrm{p}+{ }^{18} \mathrm{Ne}$ and ${ }^{18} \mathrm{O}$ scattering at incident energy $(E / A)$ and excited state energy $\left(E^{*}\right)$ in $\mathrm{MeV}$, angular momentum transfer $(l)$, deformation parameter $(\delta)$, volume integral $(J / A)$ of the real folded potential in $\mathrm{MeV} \mathrm{fm} \mathrm{fm}^{3}$ and $\chi^{2} / \mathrm{N}$ values from best-fits to the elastic and inelastic scattering data

\begin{tabular}{|c|c|c|c|c|c|c|c|c|c|c|c|c|c|}
\hline Nucleus & $E / A$ & $E^{*}$ & $\overline{N_{\mathrm{R}}}$ & $\overline{N_{\mathrm{I}}}$ & $r_{\mathrm{rms}}^{V}$ & $N_{\mathrm{R}}^{\mathrm{FF}}$ & $N_{\mathrm{I}}^{\mathrm{FF}}$ & $l$ & $\delta$ & $\overline{\chi_{\mathrm{el}}^{2} / \mathrm{N}}$ & $\chi_{\text {inel }}^{2} / \mathrm{N}$ & $J / A$ & Interaction \\
\hline${ }^{18} \mathrm{Ne}^{*}$ & 30.0 & 1.890 & 0.67 & 0.000 & 3.462 & 2.320 & 0.000 & 2 & 0.436 & 5.992 & 0.973 & -336.1 & $\overline{\mathrm{SBM}}$ \\
\hline${ }^{18} \mathrm{Ne}^{*}$ & 30.0 & 1.890 & 0.62 & 0.000 & 3.538 & 2.194 & 0.000 & 2 & 0.400 & 5.798 & 0.859 & -358.0 & DDM3Y \\
\hline${ }^{18} \mathrm{O}^{*}$ & 24.5 & 1.982 & 0.87 & 0.006 & 3.547 & 3.086 & 0.020 & 2 & 0.400 & 7.131 & 4.471 & -502.7 & $\overline{\mathrm{SBM}}$ \\
\hline${ }^{18} \mathrm{O}^{*}$ & 24.5 & 1.982 & 0.80 & 0.006 & 3.508 & 2.806 & 0.019 & 2 & 0.367 & 6.704 & 7.696 & -469.2 & DDM3Y \\
\hline
\end{tabular}


[1] K. Amos, P. J. Dortmans, H. V. von Geramb, S. Karataglidis, Adv. in Nucl. Phys. 25 (2000) 275.

[2] D. Gupta, C. Samanta, Jour. Phys. G: Nucl. Part. Phys. 28, 85 (2002); see references therein

[3] I. Tanihata, H. Hamagaki, O. Hashimoto, Y. Shida, N. Yoshikawa, K. Sugimoto, O. Yamakawa, T. Kobayashi, N. Takahashi, Phys. Rev. Lett. 55 (1985), 2676.

[4] D. Gupta, C. Samanta, R. Kanungo, Nucl. Phys. A 674 (2000), 77; see references therein

[5] L. A. Riley, J. K. Jewell, P. D. Cottle, T. Glasmacher, K. W. Kemper, N. Alamanos, Y. Blumenfeld, J. A. Carr, M. J. Chromik, R. W. Ibbotson, F. Marechal, W. E. Ormand, F. Petrovich, H. Scheit, and T. Suomijarvi, Phys. Rev. Lett. 82, 4196 (1999).

[6] J. L. Escudie, R. Lombard, M. Pignanelli, F. Resmini, and A. Tarrats, Phys. Rev. C 10, 1645 (1974).

[7] F. Petrovich, S. K. Yoon, M. J. Threapleton, R. J. Philpott, J. A. Carr, Nucl. Phys. A 563 (1993), 387.

[8] H. Rebel, G. Hauser, G. W. Schweimer, G. Nowicki, W. Wiesner and D. Hartmann, Nucl. Phys. A 218 (1974), 13.

[9] C. Samanta, Y. Sakuragi, M. Ito, and M. Fujiwara, J. Phys. G: Nucl. Part. Phys. 23 (1997), 1697; see references therein.

[10] G. R. Satchler and W. G. Love, Phys. Rep. 55 (1979), 183.

[11] D. Bandyopadhyay, C. Samanta, and S. K. Samaddar and J. N. De, Nucl. Phys. A 511 (1990), 1.

[12] C. Samanta, D. Bandyopadhyay and J. N. De, Phys. Lett. B 217 (1989), 381.

[13] R. Kanungo, C. Samanta, Subinit Roy, S. K. Samaddar, Nucl. Phys.A 581 (1995), 294.

[14] R. Kanungo, and C. Samanta, Nucl. Phys. A 617 (1997), 265; see references therein.

[15] R. Kanungo, C. Samanta, J. Phys. G: Nucl. Part. Phys. 24 (1998), 1611.

[16] R. Kanungo, I. Tanihata, C. Samanta, Prog. of Theo. Phys. 102 (1999), 1133.

[17] G. Bertsch, J. Borysowicz, H. McManus and W.G. Love, Nucl. Phys. A 284, 399 (1977).

[18] A.M. Kobos, B.A. Brown, R. Lindsay and G.R. Satchler, Nucl. Phys. A 425, 205 (1984).

[19] A.K. Chaudhuri, Nucl. Phys. A 449, 243 (1986).

[20] D. N. Basu, Jour. Phys. G: Nucl. Part. Phys. 30, B7 (2004)

[21] J. Bartel, P. Quentin, M. Brack, C. Guet, H. B. Hakansson, Nucl. Phys. A 386, 79 (1982)

[22] M. Beiner, H. Flocard, N. V. Giai, P. Quentin, Nucl. Phys. A 238, 29 (1975)

[23] N. Alamanos, A. Pakou, A. Lagoyannis, A. Musumarra, Nucl. Phys. A 660, 406 (1999)

[24] P. D. Kunz, computer code DWUCK4, unpublished

[25] B. Sinha, Phys. Rev. Lett. 50, 91 (1983)

[26] S. Raman et.al., Atomic Data and Nuclear Data Tables 36, 1 (1987)

[27] D. T. Khoa, E. Khan, G. Colo, N. Van Giai, Nucl. Phys. A 706, 61 (2002) 


\section{APPENDIX}

This appendix is aimed at providing a simple co-ordinate transformation formula for general purpose use in extracting data from plots using computer graphics.

In many instances the values of the experimental data are not available in literatures while the plots are shown. Particularly, for old plots, tabulated data are often very difficult to acquire. In such cases, data values can be read in quite accurately by using computer programs. In some cases both linear and logarithmic scales are involved. A general formula is thus required which will convert the co-ordinates of the data points as shown by the software to their actual values. It should also be taken into account that the co-ordinate system of the printed graph may be rotated, albeit small, with respect to the software co-ordinate system. Thus a handy formula would be extremely useful for such purposes.

We assume $(X, Y)$ to be the co-ordinates of the printed graph while $(x, y)$ are those of the computer program such as GSview. In Fig. 5, $\left(x_{0}, y_{0}\right)$ is the origin of the printed graph while $\left(x_{1}, y_{1}\right)$ and $\left(x_{2}, y_{2}\right)$ are two points on the $X$ and $Y$ axes, respectively.

Since the ratio of two linear lengths measured in one co-ordinate system should be equal to that in the other system, $\frac{X-X_{0}}{X_{1}-X_{0}}=\frac{\left(x-x_{0}\right) \sec \theta+\left[\left(y-y_{0}\right)-\left(x-x_{0}\right) \tan \theta\right] \sin \theta}{\left(x_{1}-x_{0}\right) \sec \theta}$ where, $\tan \theta=\frac{y_{1}-y_{0}}{x_{1}-x_{0}}$. A little manipulation gives,

$$
X=X_{0}+\left(X_{1}-X_{0}\right)\left[\frac{\left(x-x_{0}\right) \cos \theta+\left(y-y_{0}\right) \sin \theta}{\left(x_{1}-x_{0}\right) \sec \theta}\right]
$$

We consider $\mathrm{X}$ scale to be linear and Y scale to be logarithmic. Therefore, $\frac{\log Y-\log Y_{0}}{\log Y_{2}-\log Y_{0}}=\frac{\left[\left(y-y_{0}\right)-\left(x-x_{0}\right) \tan \theta\right] \cos \theta}{\left(y_{2}-y_{0}\right) \sec \theta}$ which gives,

$$
Y=Y_{0}\left(\frac{Y_{2}}{Y_{0}}\right)^{\frac{-\left(x-x_{0}\right) \sin \theta+\left(y-y_{0}\right) \cos \theta}{\left(y_{2}-y_{0}\right) \sec \theta}}
$$

If both axes are linear, then Eqn. 11 holds while

$$
Y=Y_{0}+\left(Y_{2}-Y_{0}\right)\left[\frac{-\left(x-x_{0}\right) \sin \theta+\left(y-y_{0}\right) \cos \theta}{\left(y_{2}-y_{0}\right) \sec \theta}\right]
$$

If both axes are logarithmic then Eqn. 12 holds while,

$$
X=X_{0}\left(\frac{X_{1}}{X_{0}}\right)^{\frac{\left(x-x_{0}\right) \cos \theta+\left(y-y_{0}\right) \sin \theta}{\left(x_{1}-x_{0}\right) \sec \theta}}
$$

The expressions provided above, though simple, will be immensely useful for experimentalists as well as theoreticians for extracting data where their explicit values are not available. 


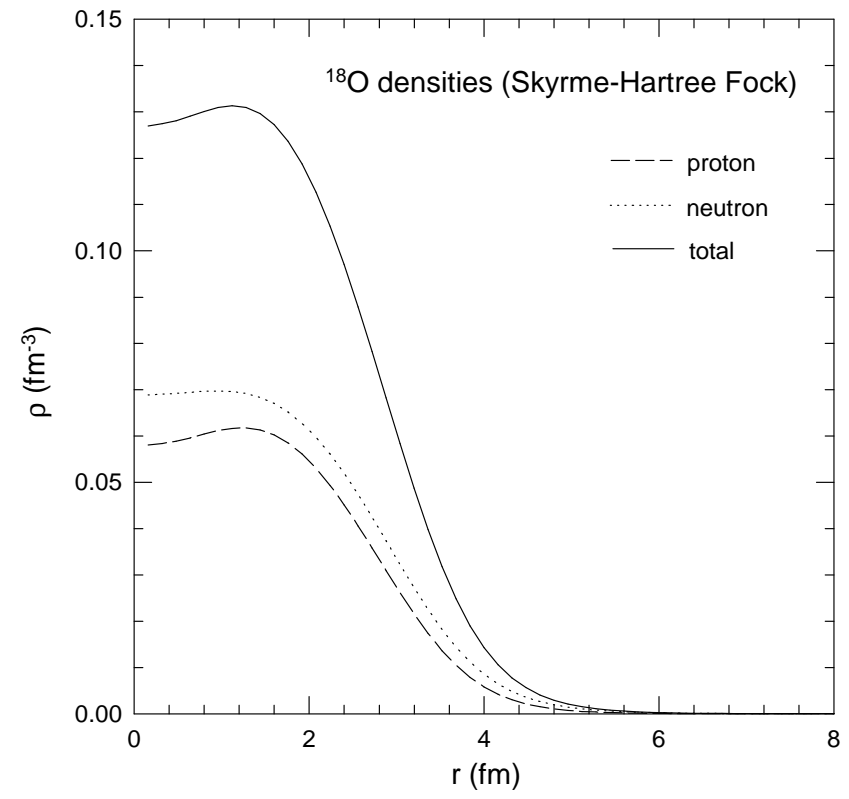

FIG. 1. Skyrme Hartree-Fock densities of ${ }^{18} \mathrm{O}$ used in this work. 


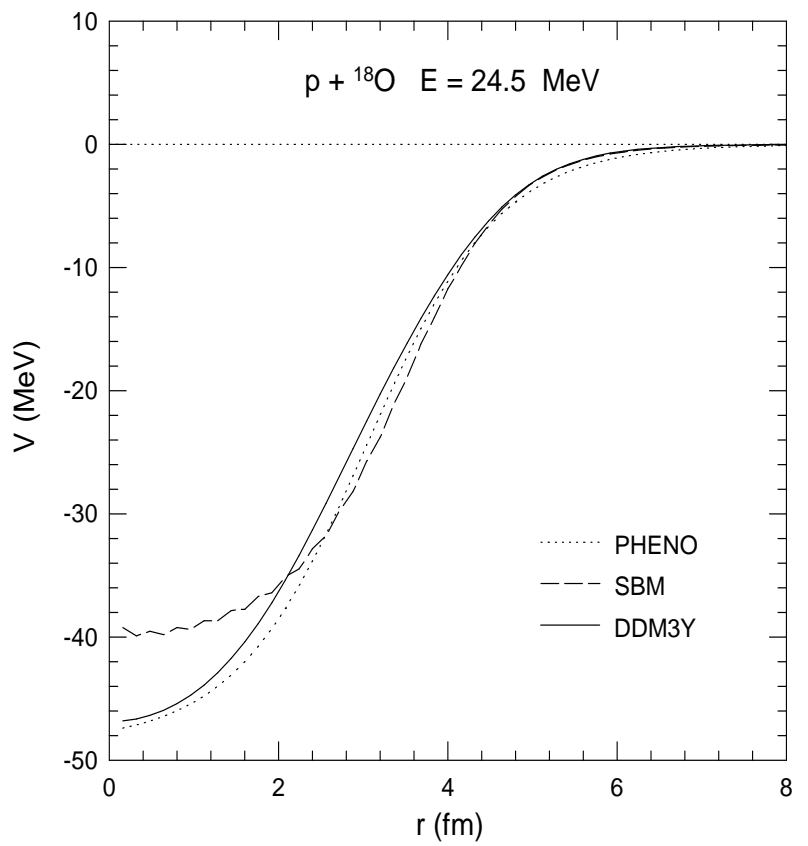

FIG. 2. The phenomenological and renormalized real folded potentials (employing SBM and DDM3Y interactions) for $\mathrm{p}+$ ${ }^{18} \mathrm{O}$ at $24.5 \mathrm{~A} \mathrm{MeV}$. 


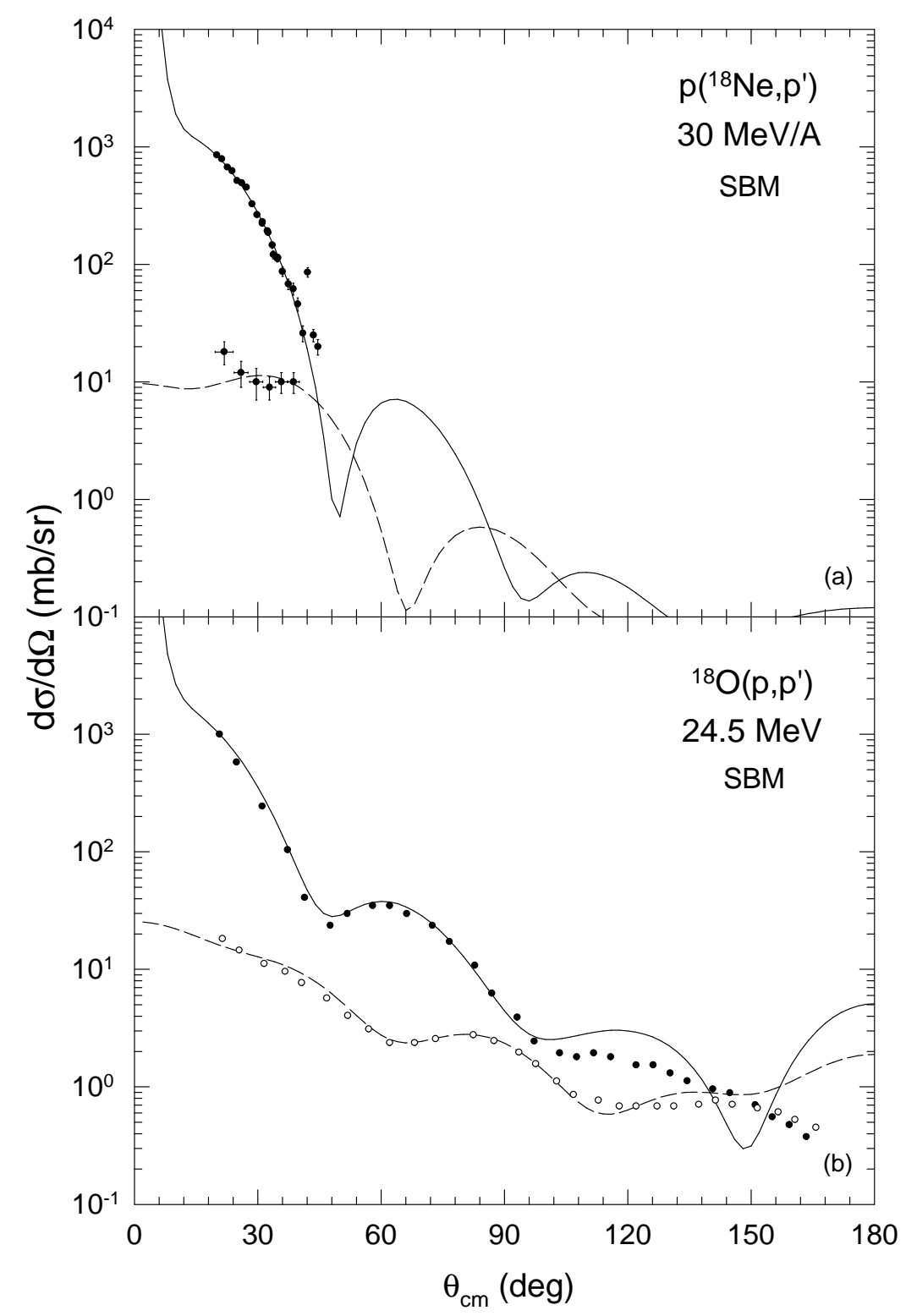

FIG. 3. The experimental angular distributions and folding model calculations (SBM) of (a) p $+{ }^{18} \mathrm{Ne}$ at $30 \mathrm{~A}$ MeV for elastic and inelastic $\left[E^{*}=1.890 \mathrm{MeV}\left(2^{+}\right)\right]$scattering [5], (b) p $+{ }^{18} \mathrm{O}$ at $24.5 \mathrm{~A} \mathrm{MeV}$ for elastic and inelastic $\left[E^{*}=1.982\right.$ $\mathrm{MeV}\left(2^{+}\right)$] scattering [6]. The corresponding $N_{\mathrm{R}}, N_{\mathrm{I}}, N_{\mathrm{R}}^{\mathrm{FF}}, N_{\mathrm{I}}^{\mathrm{FF}}$ values and phenomenological surface imaginary and spin-orbit parameters are given in Tables 3, 4. The continuous and dashed lines correspond to calculations for elastic and inelastic cross sections respectively. 


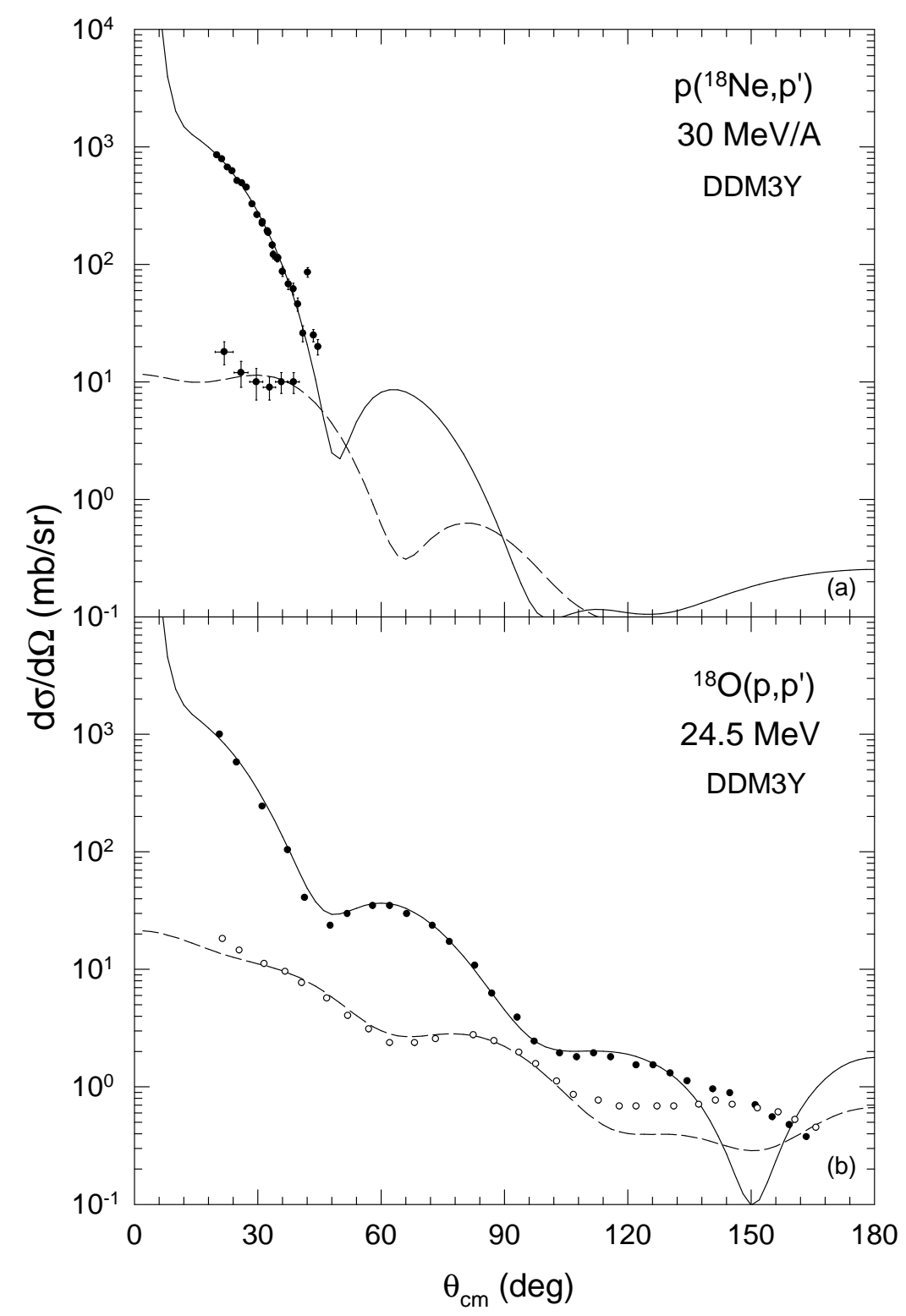

FIG. 4. The same as in Fig. 3 but employing DDM3Y interaction. 


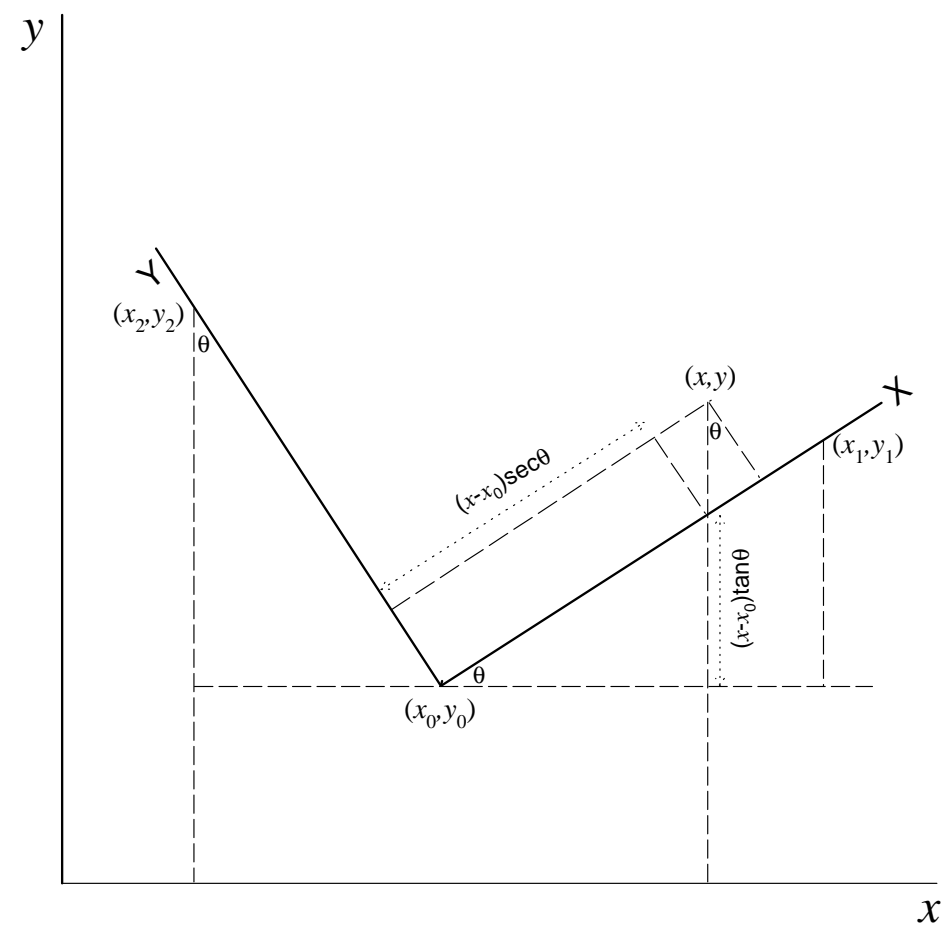

FIG. 5. $(X, Y)$ are the printed graph co-ordinate system, rotated by an angle $\theta$ with respect to $(x, y)$, the computer program co-ordinate system. 